Acta Crystallographica Section D

Biological

Crystallography

ISSN 0907-4449

D. A. R. Sanders, ${ }^{a}$

S. A. McMahon, ${ }^{a}+$ G. L. Leonard ${ }^{b}$ and J. H. Naismith ${ }^{\mathrm{a} *}$

${ }^{a}$ Centre for Biomolecular Sciences, The University, St Andrews KY16 9ST, Scotland, and ${ }^{\mathbf{b}}$ Joint Structural Biology Group, BP-220, ESRF, Grenoble CEDEX F-38043, France

+ Current address: Department of Chemistry, University of California at San Diego, 9500 Gilman Drive, La Jolla, CA 92093-0314, USA.

Correspondence e-mail: naismith@st-andrews.ac.uk

\title{
Molecular placement of experimental electron density: a case study on UDP-galactopyranose mutase
}

The structure of UDP-galactopyranose mutase, the enzyme responsible for the conversion of UDP-galactopyranose to UDP-galactofuranose, has been solved. The structure solution required the use of two crystal forms and a selenomethionine variant. Crystal form $P 2_{1}$ was used to collect a complete MAD data set, a native data set and a single-wavelength nonisomorphous selenomethionine data set. A starting set of MAD phases was then improved by non-crystallographic averaging and cross-crystal averaging of all $P 2_{1}$ data. The initial maps were of such low quality that transformation matrices between cells could not be determined. It was therefore assumed that although there were large changes in unit-cell parameters, the molecule occupied the same position in each cell. This starting assumption was allowed to refine during the averaging procedure and did so satisfactorily. Despite a visible increase in the quality of the map allowing some secondary-structural elements to be located, the overall structure could not be traced and refined. The rediscovery of the second crystal form, $P 2{ }_{1} 2_{1} 2_{1}$, allowed the collection of a native data set to $2.4 \AA$. Molecular placement of electron density was used to determine the relationship between the two unit cells. In this study, only the already averaged $P 2_{1}$ experimental density could be placed in the $P 2_{1} 2_{1} 2_{1}$ map. Less extensively density-modified maps did not give a clear solution. The study suggests even poor non-isomorphous data can be used to significantly improve map quality. The relationship between $P 2_{1}$ and $P 2_{1} 2_{1} 2_{1}$ could then be used in a final round of cross-crystal averaging to generate phases. The resulting map was easily traced and the structure has been refined. The structure sheds important light on a novel mechanism and is also a therapeutic target in the treatment of tuberculosis.

\section{Introduction}

We have previously reported the purification and crystallization of mutase from Escherichia coli (McMahon et al., 1999). Mutase initially crystallized in two different forms, only one of which was reproducible. These crystals (in the $P 2_{1}$ space group) were small two-dimensional diamond-shaped crystals that proved very difficult to work with, as they diffracted poorly and were highly mosaic. This paper describes the rediscovery of the second crystal form of mutase (in the $P 22_{1} 2_{1} 2_{1}$ space group). We detail the approach required to solve the novel structure of $E$. coli mutase, utilizing the CCP4 programs (Collaborative Computational Project, Number 4, 1994) AMoRe (Navaza, 1994), DM and DM_Multi (Cowtan, 1994) to place electron density into the new cell and determine the phases.
Received 7 February 2001 Accepted 13 June 2001
(C) 2001 International Union of Crystallography Printed in Denmark - all rights reserved 


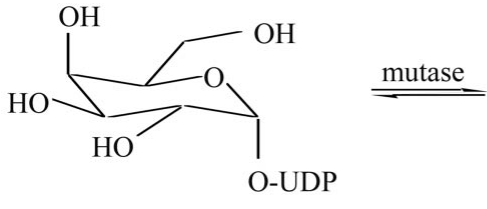

UDP-galactopyranose

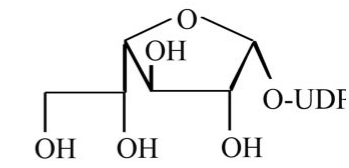

UDP-galactofuranose (Galf)

Figure 1

The reaction catalysed by UDP-galactopyranose mutase is unprecedented in biochemistry. The interconversion between the two ring forms involves no net redox reaction, although a flavin is involved.

Our laboratory has studied this protein because it is vital to the formation of the cell wall and or the capsule in many pathogenic bacteria. The biosynthesis of the cell wall is an attractive target for structure-assisted drug design of novel antibacterial drugs mainly because there are a large number of compounds in the cell wall that are not found in mammalian and other higher organisms. One such compound is galactofuranose $(\mathrm{Gal} f)$. Galf is a component of the LPS in many Gram-negative bacteria, found in the $\mathrm{O}$ antigens of many species including Klebsiella pneumonia (Koplin et al., 1997) and E. coli (Nassau et al., 1996). Galactofuranosyl residues are also an important component of the arabino-galactan complex that forms part of the mycolitic layer in the cell walls of mycobacteria (Weston et al., 1998). Gal $f$ is incorporated into cell walls from UDP-Gal $f$, formed by the enzyme UDPgalactopyranose mutase (mutase) from UDP-galactopyranose (Galp) (Fig. 1; Weston et al., 1998). The chemistry involved in this ring-contraction mechanism is completely unprecedented and is the source of great interest.

\section{Results}

\subsection{Protein purification}

The purification of E. coli mutase has been described previously (McMahon et al., 1999). Production of the SeMet

derivative protein was carried out by transfection of the $\mathrm{Met}^{-}$ strain B834 (Novagen) with the plasmid containing the E. coli gene (Nassau et al., 1996) and cell growth and induction of protein production were carried out in essentially the same manner as the native. Purification of the SeMet protein was as described for the native protein, with $2 \mathrm{~m} M$ fresh DTT in all buffers and solutions.

\subsection{Crystallization}

2.2.1. P2 1 crystal form. Crystals in the $P 2_{1}$ form were grown in $20 \%$ PEG $4 \mathrm{~K}, 12 \%$ 2-propanol, $0.01 M$ L-cysteine, $100 \mathrm{mM}$ HEPES pH 7.6 and $8 \mathrm{mg} \mathrm{ml}^{-1}$ protein as described previously (McMahon et al., 1999). SeMet protein crystals were grown in an identical manner to the wild-type protein, yielding smaller crystals than the wild type. A key point was that several seeding steps were required to obtain crystals of sufficient size for diffraction. This protocol was extremely reproducible.

2.2.2. Re-obtaining the $P 2_{1} 2_{1} 2_{1}$ crystal form. A solitary single crystal was obtained in the space group $P 2_{1} 2_{1} 2_{1}$ form and this was originally described in McMahon et al. (1999). We have been unable to reproduce this crystal. The initial crystal was grown from an early purification run of mutase and had impurities in it. Subsequent purifications resulted in homogenous protein. Deliberately 'contaminated' protein did not crystallize. However, by the streak-seeding of $P 2_{1}$ crystals into drops containing 10-20\% PEG 6K, $100 \mathrm{~m} M$ HEPES pH 7.0,

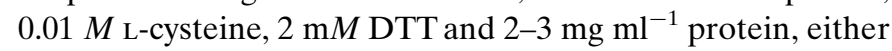
pre-equilibrated overnight (for the $10 \%$ drops) or seeded immediately (for the $20 \%$ drops), we were successful in growing crystals in the $P 2_{1} 2_{1} 2_{1}$ form. These crystals grew overnight to $0.2 \times 0.2 \times 0.1 \mathrm{~mm}$ in size. Repeated seedings and macroseeding with single crystals generally failed to increase the size.

\section{Table 1}

Summary of crystallographic data.

Values in parentheses refer to the highest resolution shell.

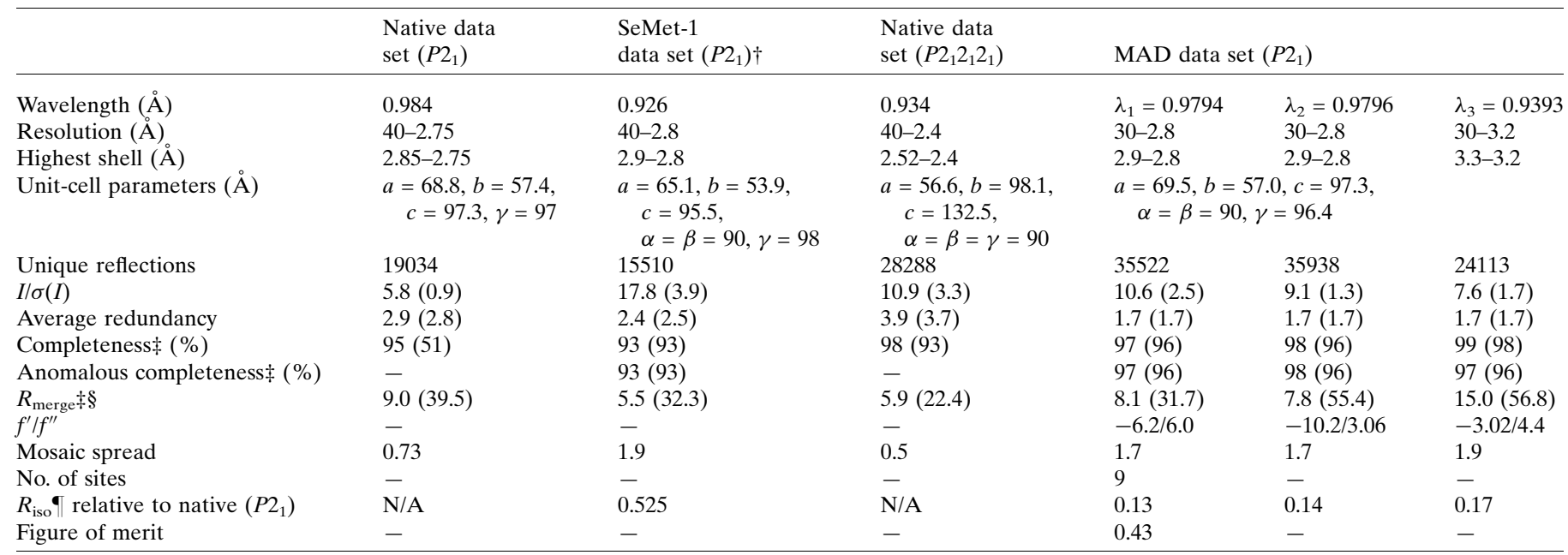

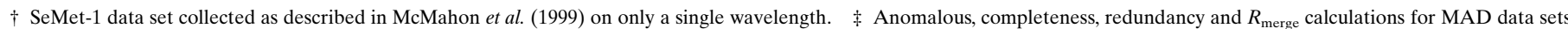

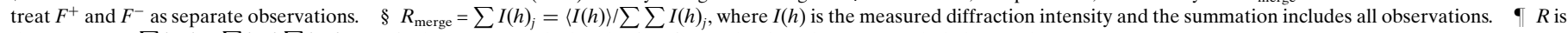
the $R$ factor $=\sum\left|F_{o}\right|-\sum\left|F_{c}\right| / \sum\left|F_{o}\right| . R_{\text {free }}$ is the $R$ factor calculated using $5 \%$ of the data that were excluded from the refinement. 


\subsection{Data collection}

2.3.1. P2 ${ }_{1}$ data. The SeMet-1 data set to $3.0 \AA$ was collected as described previously (McMahon $e t$ al., 1999). The native and SeMet-2 data sets for the $P 2_{1}$ crystal form were collected on beamline ID14-4 at the ESRF synchrotron in Grenoble. Although crystals were generally highly variable in diffraction and had high mosaic spread, several crystals of sufficient quality were found to collect the necessary data sets. Owing to the fragile nature of the crystals, we used a poor-quality larger crystal to EXAFS scan in order to determine the three wavelengths for the MAD data set. The native data set consisted of 180 nonoverlapping $1^{\circ}$ images collected for $30 \mathrm{~s}$ to $2.7 \AA$ and the SeMet data set was to $2.8 \AA$ resolution for two wavelengths and $3.2 \AA$ for the third (Table 1), each consisting of $1801^{\circ}$ non-overlapping images collected for $15 \mathrm{~s}$. Data were processed using the programs MOSFLM (Leslie, 1992) and SCALA (Evans, 1997), treating the Bijvoet pairs as independent measurements for the SeMet data set.

2.3.2. $P 2_{1} 2_{1} 2_{1}$ data. The data for the $P 2_{1} 2_{1} 2_{1}$ crystal form were collected on beamline ID14-1 at the ESRF synchrotron in Grenoble. A single crystal of about $0.15 \times 0.15 \times 0.075 \mathrm{~mm}$ in size had to be cryoprotected in a relatively complex manner. The crystal was moved (using a glass capillary $\sim 0.2 \mathrm{~mm}$ size) into a stabilizing buffer $(25 \%$ PEG $4 \mathrm{~K}$, 15\% 2-propanol, $100 \mathrm{~m} M$ HEPES $\mathrm{pH}$ 7.6) and after $\sim 1$ min was moved rapidly through stabilizing buffer plus $7.5 \%$ glycerol into stabilizing buffer plus $15 \%$ glycerol, in which it was then frozen. The initial image of this crystal showed high mosaicity. The mosaicity was significantly reduced by a round of reannealing/flash-freezing by blocking the Cryostream for $5 \mathrm{~s}$. A data set was collected to $2.4 \AA$ resolution,
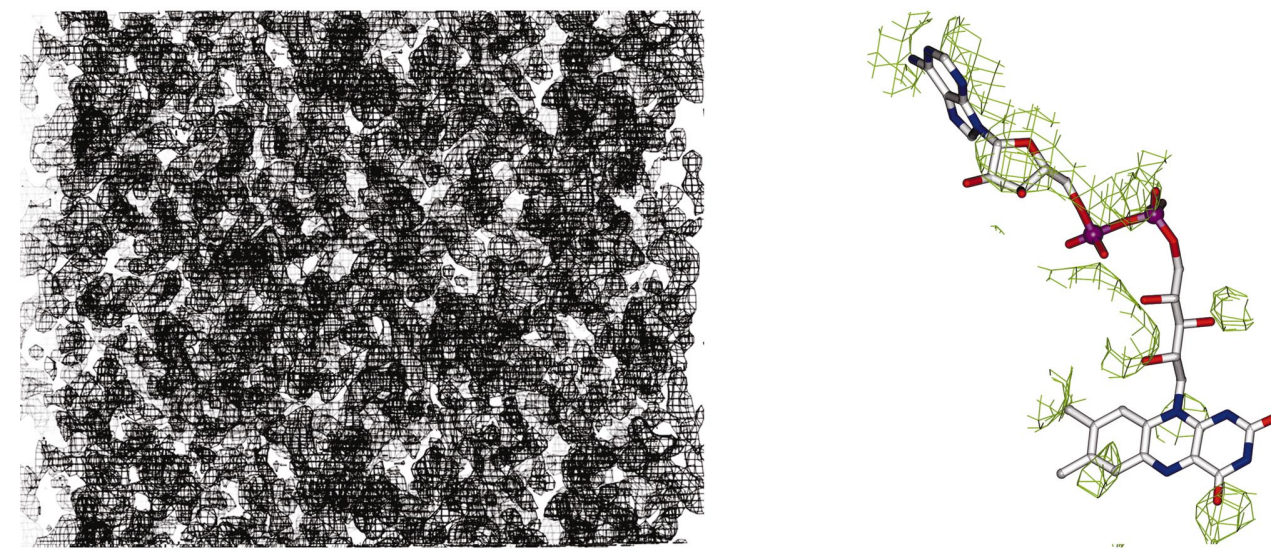

(a)
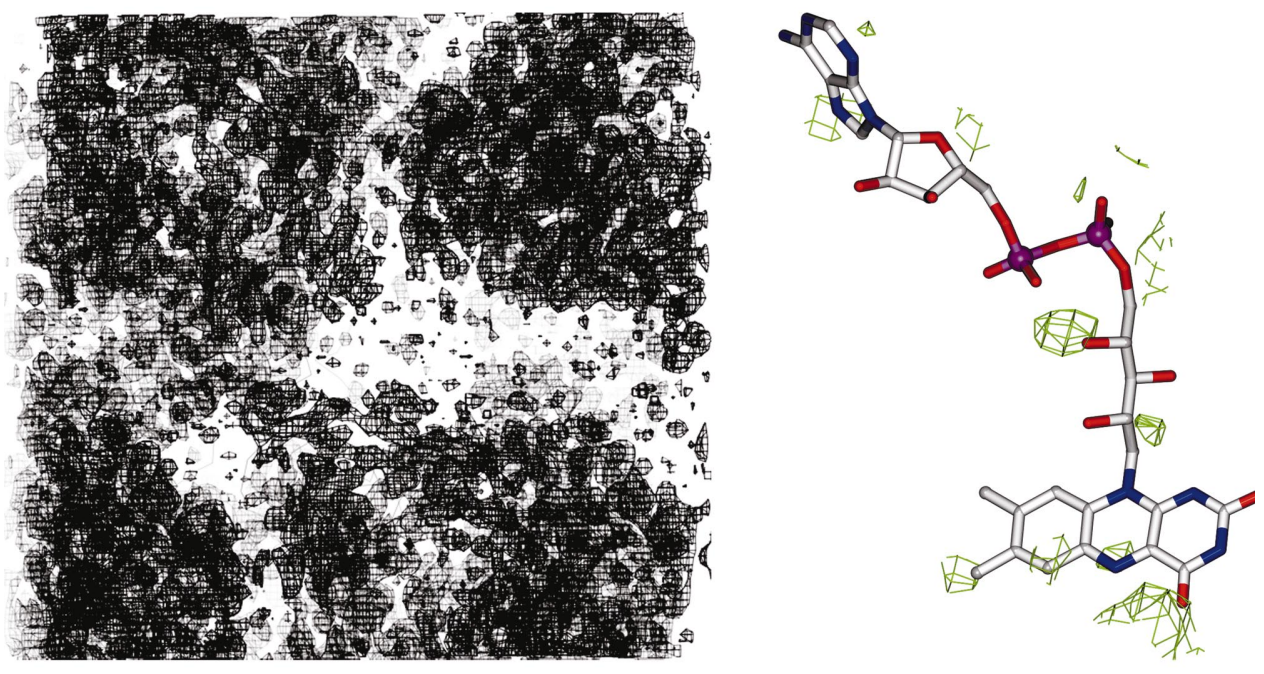

(b)
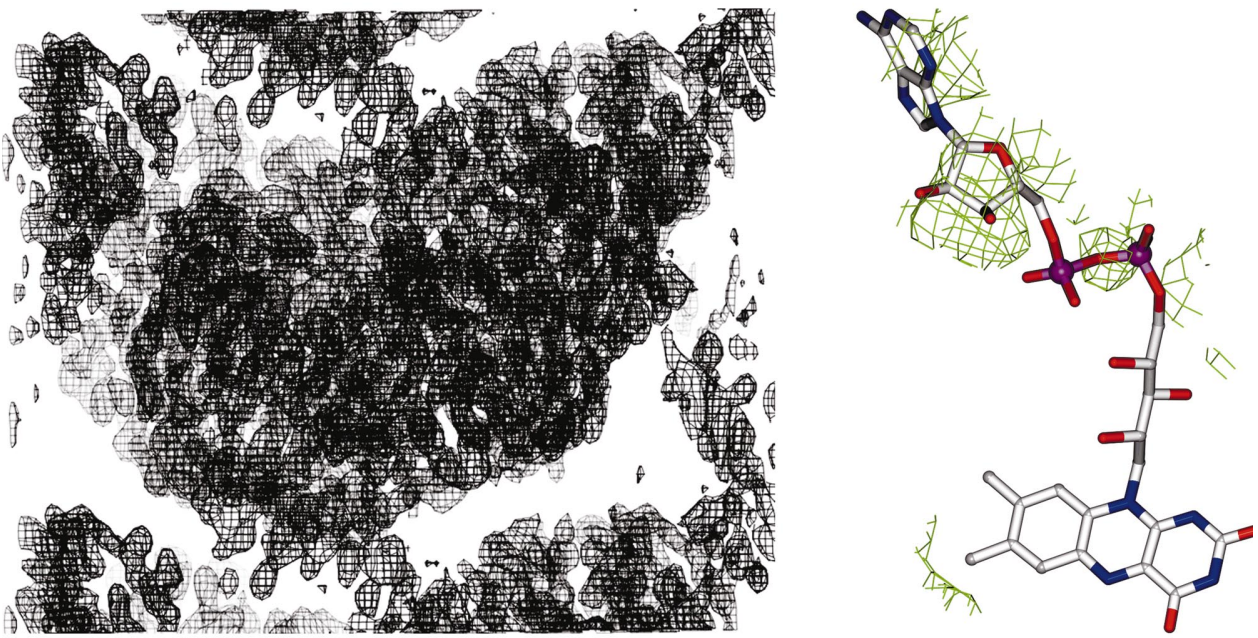

(c)

Figure 2

Experimental electron density contoured at $1 \sigma$. On the left is density of the same $75 \AA^{2}$ region of space, while on the right is a stereoview of FAD with the density from the map. Figures generated with Snapshot from $O$ (Jones et al., 1991) (left) and BOBSCRIPT (Esnouf, 1997) utilizing the GL_RENDER interface (L. Esser and J. Deisenhofer, unpublished work) and POV-Ray (right). (a) Initial map from SOLVE. (b) Map after initial solvent flattening, averaging and phase extension. (c) Map after mask improvement, crosscrystal averaging with native, SIROAS and non-isomorphous SeMet-1 data. Back-translation of the final refined structure into the $P 2_{1}$ cell shows that the solvent boundaries in $(b)$ are completely wrong. 
consisting of $2200.5^{\circ}$ non-overlapping images collected for $30 \mathrm{~s}$ each. Data were processed using MOSFLM and SCALA (details are given in Table 1).

\subsection{Structure solution}

2.4.1. P2 1 solution. The program SOLVE 1.17 (Terwilliger \& Berendzen, 1999) was used to locate the selenium sites in the $P 2_{1}$ crystal form. Nine of a possible 14 sites were found $(Z$ score $=20, \mathrm{FOM}=0.43)$. Eight of these sites were consistent with a twofold rotation suggested by the selfrotation map. The initial map was improved by solvent flattening with $D M$ (Cowtan, 1994) with phase extension to $2.7 \AA$. As shown in Fig. 2(b), the map was very poor and no convincing evidence of secondary structure was found. Close analysis of the data indicated that the anomalous signal from the SeMet data was very weak. One indicator of the poor quality of the anomalous signal is the ratio $\Delta_{\text {ano }} / \sigma\left(\Delta_{\text {ano }}\right)$, which in the case of the mutase data was significantly less than one for all three wavelengths $(0.67,0.62$ and 0.58 for the peak, inflection and remote wavelengths, respectively). SOLVE also indicated the same problem. Some additional 'improvement' of the phases was made by placing a $50 \AA$ sphere as a mask centred at the centroid of the selenium positions. This mask was improved by manually rebuilding the map to fill areas of density through visual inspection.

MLPHARE (Otwinowski, 1991) was used to generate SIROAS phases using the peak wavelength for SeMet-2 against the native data set. Consistent with the weak signal, the anomalous occupancy tended to zero when refined in MLPHARE. We then began cross-crystal averaging between the native (SIROAS phases), SeMet-2 (MAD phases) and SeMet-1 data. A monomer mask derived from the earlier $50 \AA$ mask was used as the NCS mask. The translation matrices for these data sets were assumed to be unity and allowed to refine in the program. This is despite effectively complete nonisomorphism between SeMet-1 and the two other data sets; the average mean fractional isomorphous difference was 0.525 . Attempts to improve the matrices prior to DMMULTI
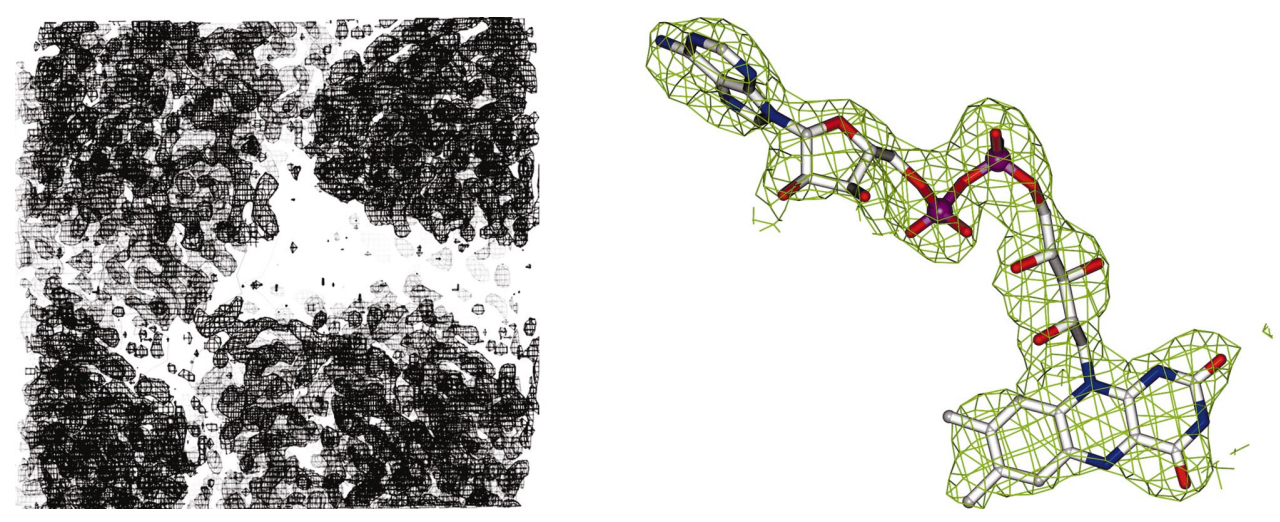

Figure 3

Final experimental density map for the $P 2_{1} 2_{1} 2_{1}$ data. Density is contoured at $1 \sigma$ and is of the same region of space as in Fig. 2. This figure is of a different cell $\left(P 2_{1} 2_{1} 2_{1}\right)$ compared with Fig. $2\left(P 2_{1}\right)$ and so cannot be directly compared.
Table 2

$A M o R e$ results for molecular replacement using density.

\begin{tabular}{lrllllllll}
\hline Solution & \multicolumn{1}{c}{$a$} & $b$ & $c$ & $x$ & \multicolumn{1}{c}{$y$} & \multicolumn{3}{c}{$\begin{array}{l}\text { Corr. } \\
\text { coeff. }\end{array}$} & $\begin{array}{l}R \\
\text { factor }\end{array}$ \\
\hline Solution 1 & 41.09 & 86.47 & 337.52 & 0.1623 & 0.1780 & 0.0908 & 24.7 & 52.2 \\
Solution 2 & 91.90 & 82.46 & 182.15 & 0.0902 & 0.2498 & 0.000 & 20 & 54.6 \\
Solution 3 & 137.23 & 90.00 & 156.92 & 0.3437 & 0.1806 & 0.4177 & 19.3 & 53.5 \\
Solution 4 & 26.96 & 43.32 & 35.55 & 0.9988 & 0.2981 & 0.0330 & 8.3 & 57.5 \\
& & & & & & & & \\
$\begin{array}{l}\text { Solution 1 } \\
\quad \text { after fitting }\end{array}$ & 41.02 & 86.67 & 337.85 & 0.1630 & 0.1756 & 0.0908 & 26.1 & 51.9 \\
\hline
\end{tabular}

by density-placement calculations (Cowtan, 1994) gave worse results (as measured by correlation coefficients and visual examination of maps). The results from this DMMULTI run were promising, with a final correlation coefficient for monomer $A$ between native and SeMet-1 of 0.935 and an FOM of 0.66 for $2.8 \AA P 2_{1}$ native data. This map was the used for an initial model-building step. Clear secondary-structure elements (mostly $\alpha$-helices with a few $\beta$-strands) were visible for the first time in the map. The map is shown in Fig. 2(c). A polyalanine model consisting of 300 residues was traced into this map, possible secondary-structure elements were assigned and this model was used to search DALI (Holm \& Sanders, 1993) and DEJAVU (Kleywegt \& Jones, 1997) for similar proteins; however, no solutions were found. An attempt was made to refine this model using CNS (Brunger et al., 1998), but the model did not refine.

2.4.2. $P 2{ }_{1} 2_{1} 2_{1}$ solution. At the point where the $P 2_{1}$ data failed to render a solution, the $P 2_{1} 2_{1} 2_{1}$ crystal form was rediscovered and the data was collected. The transformation matrix between the two crystal forms was generated by using molecular placement of the cross-crystal averaged electron density from $P 2_{1}$. The $P 2_{1}$ model was used to create a mask of the monomer inside the $P 2_{1}$ unit cell (using MAPMASK; Collaborative Computational Project, Number 4, 1994). This mask was then applied to the 'final' map from the same solution $P 2_{1}$ solution (NCS, cross-crystal averaged) (using MAPMASK and OVERLAPMASK; Jones \& Stuart, 1991). The region outside of the mask was flattened and the resulting density was transformed to a $P 1$ symmetry cell (EXTEND; Collaborative Computational Project, Number 4, 1994). This map was then back-transformed using SFALL (Collaborative Computational Project, Number 4, 1994) to generate the structure factors, which were normalized to $E$ values (ECALC; Collaborative Computational Project, Number $4,1994)$ and ordered for use in $A M o R e$ (Navaza, 1994) by $C A D$ (Collaborative Computational Project, Number 4, 1994). The calculated data were sorted and tabled in $A M o R e$ and then run against the $P 22_{1} 2_{1} 2_{1}$ data to find 
Table 3

Results of DMMULTI cross-crystal averaging between $P 2_{1}$ and $P 2_{1} 2_{1} 2_{1}$ crystal forms.

\begin{tabular}{llllll}
\hline & \multicolumn{2}{l}{ Correlation coefficient } & & FOM & \\
\cline { 2 - 3 } Data set & Before $D M \dagger$ & After $D M \dagger$ & & Before & After \\
\hline$P 2_{1}$ native & $-/ 0.19$ & $-/ 0.92$ & & 0.13 & 0.62 \\
SeMet-1 & $0.00 / 0.03$ & $0.88 / 0.86$ & & 0.00 & 0.61 \\
$P 2_{1} 2_{1} 2_{1}$ & $0.01 / 0.01$ & $0.84 / 0.80$ & & 0.00 & 0.48 \\
\hline
\end{tabular}

$\dagger$ Each crystal has two monomers, monomer $A$ and monomer $B$. The starting point for averaging is monomer $A$ of $P 2_{1}$ native. The values refer to the correlation between $P 2_{1}$ monomer $A$ and monomer $A$ /monomer $B$ of the other crystals.

solutions for the monomer (see Table 2). The matrix between the two monomers in the $P 2_{1} 2_{1} 2_{1}$ unit cell was derived by applying the $A M o R e$ monomer solution to the positions of the Se atoms determined from SOLVE. The relationship between the two sets of Se atoms determined the matrix between the monomers. We based this on the assumption that the position of the two monomers did not change between the two different crystal forms. We then used cross-crystal averaging (DMMULTI; Cowtan, 1994) using this transformation and four data sets $\left[P 2_{1}\right.$ native with the 'final' phases, $P 2_{1}$ SeMet-2 peak wavelength with phases from SOLVE/DM (SIROAS phases), SeMet-1 data set and the $P 2{ }_{1} 2_{1} 2_{1}$ data set]. The rotation matrix for the different $P 2_{1}$ data sets was assumed to be unity and all of the matrices were allowed to be refined by DMMULTI. Table 3 shows the starting and finishing correlation coefficients and FOMs from the final DMMULTI run. Fig. 3 shows the density of the experimental phases (prior to refining) at the position of refined FAD.
Table 4

Final refinement statistics for E. coli mutase.

\begin{tabular}{ll}
\hline No. of protein atoms & 6084 \\
No. of water molecules & 321 \\
No. of heteroatoms & 106 \\
Root-mean-square deviations from & \\
$\quad$ Ideal bond lengths $\dagger$ & 0.012 \\
$\quad$ Ideal bond angles $\dagger$ & 1.4 \\
Mean temperature factor & \\
$\quad$ Main chain & 26.55 \\
$\quad$ Side chain & 29.11 \\
$\quad$ Heteroatoms & 19.37 \\
$\quad$ Water molecules & 32.23 \\
Crystallographic $R$ factor $(\%)$ & 18.52 \\
Free crystallographic $R$ factor $¥(\%)$ & 24.52 \\
\hline
\end{tabular}

$\dagger$ R.m.s. deviation from Engh and Huber ideal values (Engh \& Huber, 1991). $\quad \neq R_{\text {free }}$ is calculated on $5 \%$ of data excluded during refinement.

At this point, a polyalanine model was built into the experimental density map, allowing tracing of 321 residues (of 367); the FAD was also found. The resulting model and known NCS operators were then used for real-space averaging $(R A V E)$. This allowed further identification of 20 residues and also allowed the joining of different segments of the structure. This model was run through DALI (Holm \& Sanders, 1993) and DEJAVU (Kleywegt \& Jones, 1997). This confirmed that the FAD-binding domain was similar to a number of other proteins; however, the second domain and linking region were novel. Refinement was begun using CNS (Brunger et al., 1998), starting with simulated annealing, rigid-body refinement and positional/ $B$-factor refinement. This allowed the location of the final 26 residues and joining of the last segments (Fig. 4). Further refinement proceeded smoothly and resulted in a model with final refinement statistics as listed in Table 4.

\section{Discussion}

The solution of the E. coli mutase structure has proven to be exceedingly difficult owing to the small size and high degree of mosaicity of the crystals. The $P 2_{1}$ crystal form with native and SeMet derivative (MAD) data sets proved insufficient to solve the phase problem, most likely owing to the very poor anomalous signal in the MAD data sets. The poor anomalous signal was probably the result of the high mosaicity and concomitant poor diffraction. Attempts to obtain heavy-metal derivatives of the $P 2_{1}$ crystal form were hampered by our inability to screen crystals in-house. Limited synchrotron time also prevented extensive screening and no useful information was obtained
Figure 4

Superposition between model built from $P 2_{1}$ data (in red, unrefinable) and final refined mutase monomer from $P 2{ }_{1} 2_{1} 2_{1}$ data (in various blues). Superposition performed initially with $L S Q K A B$ (Kleywegt \& Jones, 1997) and then adjusted manually in $O$ (Jones et al., 1991). 
with the putative platinum derivative collected at Grenoble (McMahon et al., 1999).

The discovery that the $P 2_{1} 2_{1} 2_{1}$ crystals could be grown by seeding with the $P 2_{1}$ crystal form proved instrumental in solving the structure. The initial $P 2_{1} 2_{1} 2_{1}$ crystal reported earlier was probably grown in a drop containing enough impurities to provide a nucleation site; however, subsequent attempts to return to this condition were unsuccessful owing to, ironically, greater attention to sample purity. Micro/streak seeding of $P 2_{1}$ crystals into these drops was enough to allow the $P 22_{1} 2_{1}{ }_{1}$ form to be grown. These crystal proved to be much better for diffraction purposes, despite a much more rigorous freezing protocol.

Owing to the fact that there is apparently a mathematical relationship between the two cells (as in Table 1: $a_{P 2_{1} 2_{1} 2_{1}}=b_{P 2_{1}}$, $\left.b_{P 2_{1} 2_{1} 2_{1}}=c_{P 2_{1}}, c_{P 2_{1} 2_{1} 2_{1}}=2 a_{P 2_{1}}\right)$, we began deriving the rotation matrix between the two crystal forms mathematically. The rapid success of molecular replacement using density meant that we never completed the mathematical transformation.

During a post-mortem analysis we tried to place electron density from the initial $P 2_{1}$ MAD data directly obtained from $S O L V E$. No solution was found, although careful examination of the rotation-function solutions did show one peak with approximately the correct rotation/translation solution, although the $z$-axis translation was quite different (solution $=$ $42.07,87.17,332.57,0.21,0.18,0.38)$. The same test of the density-modified map (Fig. $2 b$ ) gave similarly poor results. Experience in the laboratories suggests a good indicator of a correct solution can be found by viewing the $\mathrm{min} / \mathrm{max}$ density of the rotation map. The ratio of (max. density/r.m.s. deviation):(min. density/r.m.s. deviation) gives a fairly reliable indicator of height of peaks above noise level. In our example, the successful molecular replacement gave a ratio of 8.3:(-3.9) compared with 4.9:(-0.9) and 5.0:(-3.9) for the results from SOLVE and DM (Cowtan, 1994), respectively.

Our results confirm the adage that while correlation coefficient and FOM are useful guides toward determining whether the phases derived are correct, visual examination of maps and the appearance of such easily discernable features as $\alpha$-helices are better guides towards structure solution. We have also demonstrated that placement of electron density and cross-crystal averaging are very powerful tools for solving structures using very poor initial phases. The post-mortem analysis shows that only by extracting the maximum phase information from the SeMet-2 data (by manual adjustment of the mask and SIROAS phases from the peak wavelength) and cross-crystal averaging against the non-isomorphous SeMet-1 data set (which was very poor quality), could good enough density be obtained for placement in the $P 2_{1} 2_{1} 2_{1}$ cell.

We thank Garry Taylor for the assistance with placement of density, Kevin Cowtan for help with $D M / D M_{-}$Multi and Eleanor Dodson for many helpful suggestions and comments on solving the phase problem using many different approaches.

\section{References}

Brunger, A. T., Adams, P. D., Clore, G. M., DeLano, W. L., Gros, P., Grosse-Kunstleve, R. W., Jiang, J.-S., Kuszewski, J., Nilges, M., Pannu, N. S., Read, R. J., Rice, L. M., Simonson, T. \& Warren, G. L. (1998). Acta Cryst. D54, 905-921.

Collaborative Computational Project, Number 4 (1994). Acta Cryst. D50, 760-763.

Cowtan, K. (1994). Jnt CCP4/ESF-EACBM Newsl. Protein Crystallogr. 31, 34-38.

Engh, R. A. \& Huber, R. (1991). Acta Cryst. A47, 392-400.

Esnouf, R. M. (1997). J. Mol. Graph. Model. 15, 132-134.

Evans, P. R. (1997). Jnt CCP/ESF-EACBM Newsl. Protein Crystallogr. 33, 22-24.

Holm, L. \& Sanders, C. (1993). J. Mol. Biol. 233, 123-138.

Jones, T. A., Zou, J.-Y., Cowan, S. W. \& Kjeldgaard, M. (1991). Acta Cryst. A47, 110-119.

Jones, Y. \& Stuart, D. I. (1991). Proceedings of the CCP4 Study Weekend. Isomorphous Replacement and Anomalous Scattering, edited by W. Wolf, P. R. Evans \& A. G. W. Leslie, pp. 39-48. Warrington: Daresbury Laboratory.

Kleywegt, G. J. \& Jones, T. A. (1997). Methods Enzymol. 277, 525545.

Koplin, R., Brisson, J.-R. \& Whitfield, C. (1997). J. Biol. Chem. 272, 4121-4128.

Leslie, A. G. W. (1992). Jnt CCP4/ESF-EACMB Newsl. Protein Crystallogr. 26.

McMahon, S. A., Leonard, G. L., Buchanan, L. V., Giraud, M.-F. \& Naismith, J. H. (1999). Acta Cryst. D55, 399-402.

Nassau, P. M., Martin, S. L., Brown, R. E., Weston, A., Monsey, D., McNeil, M. R. \& Duncan, K. (1996). J. Bacteriol. 178, 1047-1052. Navaza, J. (1994). Acta Cryst. A50, 157-163.

Otwinowski, Z. (1991). Proceedings of the CCP4 Study Weekend. Isomprphous Replacement and Anomalous Scattering, edited by W. Wolf, P. R. Evans \& A. G. W. Leslie, pp. 80-86. Warrington: Daresbury Laboratory.

Terwilliger, T. C. \& Berendzen, J. (1999). Acta Cryst. D55, 849-861. Weston, A., Stern, R. J., Lee, R. E., Nassau, P. M., Monsey, D., Martin, S. L., Scherman, M. S., Besra, G. S., Duncan, K. \& McNeil, M. R. (1998). Tubercle Lung Dis. 78, 123-131. 